\title{
Impact of Sex on Cardiac Remodelling and Long-Term Outcomes Following Mitral Valve Replacement
}

\author{
Ryaan EL-Andari ${ }^{1}$, Sabin Bozso ${ }^{1}$, Jimmy Kang ${ }^{1}$, Dana Boe ${ }^{1}$, Nicholas Fialka ${ }^{1}$, Yongzhe \\ Hong $^{1}$, Michael Moon ${ }^{1}$, Darren Freed ${ }^{1}$, Jayan Nagendran ${ }^{1}$, and Jeevan Nagendran ${ }^{1}$ \\ ${ }^{1}$ University of Alberta
}

October 5, 2020

\begin{abstract}
Background: Differences in cardiac remodeling after mitral valve (MV) surgery between the sexes is poorly understood. Inferior outcomes for females undergoing MV surgery compared to males have been suggested in the literature, although causative factors behind this discrepancy have not been identified. Materials and Methods: In this propensity-matched, retrospective, singlecenter study, we sought to identify the impact that sex may have on cardiac remodeling and long-term outcomes to better inform clinical decision making in MV surgical intervention. Outcomes were compared between males and females undergoing MV replacement (MVR) between 2004 and 2018. The primary outcome was cardiac remodeling 1 year postoperatively. Secondary outcomes included mortality, stroke, myocardial infarction (MI), reoperation of the MV, and rehospitalization. Results: 314 males and 314 females were included after propensity matching. Males demonstrated a significant degree of improved left ventricular remodeling while females did not, and females showed a significant degree of left atrial remodeling while males did not. Mortality rates were relatively equivalent between the two groups, although males were more likely to develop sepsis and require rehospitalization due to MI. Conclusions: There has been little research exploring the differences in cardiac remodeling between the sexes after MVR. The results of this study have suggested that MVR is equally safe for both sexes and has demonstrated a difference in the heart's ability to remodel after MVR. The significance of this difference has the potential to result in largely different clinical outcomes for males and females. Further study is necessary to fully elucidate this relationship.
\end{abstract}

\section{Introduction}

The prevalence of valvular heart disease (VHD) is estimated to be $2.5 \%$ in developed nations and increases with age, occurring in $14.0 \%$ and $12.6 \%$ of males and females over the age of 75 , respectively [1,2]. Mitral valve disease (MVD) is the most common valvular lesion, with at least moderate mitral regurgitation (MR) occurring in $1.7 \%$ of all individuals and $9.3 \%$ of those over the age of 75 [2]. The only definitive treatment for MVD is surgical repair or replacement with a mechanical or bioprosthetic valve. Consideration for MV surgical intervention depends on the expected outcomes associated with various preoperative and intraoperative factors and sex is a factor that has been demonstrated to influence these factors $[8,14]$. Preoperatively, females are referred later in the disease process with generally higher rates of comorbidities [11-15]. Intraoperatively, there is an increased likelihood of MVR over repair for females compared to males $[12,13,15]$ and an increased likelihood of concomitant procedures [13, 14]. Postoperatively, studies have demonstrated inferior outcomes for females as compared to males in MV surgery, with up to a 2.5x increased mortality for females aged 40-59 [11, 12, 18, 19]. While it is well known that the heart remodels after MV surgery, there is little evidence on the impact of sex on cardiac remodeling post MV surgery.

Cardiac remodeling is an integral part of valvular and other cardiac diseases. Changes to hemodynamics, chamber pressure, metabolism, inflammation, and a multitude of other mechanisms can lead to remodeling of the cardiac chambers. In the context of MV disease, cardiac remodeling most often includes left atrial (LA) and left ventricular (LV) dilation as well as an increased LV ejection fraction (LVEF). The consequences of 
cardiac remodeling include development of arrhythmias and heart failure [20,21]. It is therefore imperative to understand the factors influencing cardiac remodeling after MV surgery as reversal of pathological remodeling may impact an individual's recovery and long-term prognosis.

Although higher rates of comorbidities and delayed surgical referral may explain the inequity in MV surgery outcomes between males and females, there have been no factors definitively identified as having a causal effect. The current literature seeking to identify the impact of sex and surgical approach on postoperative cardiac remodeling and outcomes is insufficient. Consequently, identical guidelines are used to treat both sexes while females continue to suffer inferior postoperative outcomes. Current trends in medicine seek to personalize treatments for individual patients. In order to minimize the disease burden and optimize quality of life, treatment in MVD should also be tailored to an individual's specific presentation. In this propensitymatched retrospective study, we look to identify variations in postoperative cardiac remodeling and outcomes between males and females who have undergone MVR to better inform the clinical decision-making process for patients requiring surgical MVR.

\section{Materials and Methods}

Patient data were collected from a database that included surgical intervention, preoperative comorbidities, as well as postoperative complications and outcomes. 2,912 patients who had undergone either a MV repair or replacement from 2004-2018 at the Mazankowski Heart Institute, University of Alberta Hospital were included in the database. Follow up information and outcomes from all post-operative assessments performed at the University of Alberta Hospital were also included. Approval for this study was obtained from the local research ethics board. Individual waiver for consent was granted by the local research ethics board.

\section{Data Source}

The APPROACH (Alberta Provincial Project for Outcome Assessment in Coronary Heart Disease) database, electronic health records, and discharge abstract databases were utilized in order to collect the relevant pre and postoperative information. The APPROACH database contains comprehensive clinical information from patients undergoing coronary angiography in Alberta. The details of this clinical database and its use have been described previously [28].

\section{Study Cohort}

This study included patients with severe MV disease that underwent MVR at the University of Alberta Hospital between January 1, 2004, and December 31, 2018 (Figure 1). Exclusion criteria included repair of the MV or if data was incomplete $(n=1673)$, if they were in a critical preoperative state $(n=24)$ (need for intra-aortic balloon pump, acute renal failure requiring dialysis, respiratory failure requiring ventilation), or if there was a preoperative diagnosis of endocarditis $(n=96)$. A total of 1072 patients were included consisting of 476 males and 596 females (Figure 1). After propensity matching, 314 males and 314 females were included. Patients were followed for a maximum of 16 years with a median follow-up of 8.4 years.

\section{Outcomes}

The primary outcome was cardiac remodeling within 1 year postoperatively. Functional, anatomical, and hemodynamic assessments included preoperative and postoperative LVEF, LV internal dimension in diastole (LVIDd), LA size (mm), LA volume index $\left(\mathrm{ml} / \mathrm{m}^{2}\right)$, MV area (MVA) $\left(\mathrm{cm}^{2}\right)$, and MV peak and mean gradients. Secondary outcomes include mortality at any time postoperatively, postoperative stroke, myocardial infarction (MI), reoperation of the MV, and rehospitalization. Both during admission and following discharge for the index procedure, outcome data were collected and identified based on admitting diagnosis for any readmission. MI was defined as the primary diagnosis of ST-segment elevation MI (STEMI) or non-STEMI for readmission any time during the follow-up period. Stroke was defined as the primary diagnosis at readmission at any point during the follow-up period. Reoperation of the MV was defined as MVR, surgical repair, or repair of complications originating from the index procedure. New-onset AF was defined as AF 
arising after the index procedure with no prior history of AF. Residual MR included moderate or severe MR within 1 year postoperatively.

\section{Statistical analysis}

Continuous variables and categorical variables were summarized as mean \pm standard deviation (SD) and count (percent) respectively. The Society of Thoracic Surgery (STS) Risk Score for each patient was calculated using the online STS calculator [29]. Propensity score matching techniques were used to control for the difference in the baseline covariates. The propensity score was estimated using a multivariable logistic regression model with biological sex as the dependent variable and the baseline characteristics as covariates including age, BMI, pulmonary disease, cerebrovascular disease, renal disease, current smoker, past smoker, hypertension, dyslipidemia, liver disease, gastrointestinal disease, malignancy, peripheral vascular disease, diabetes mellitus, congestive heart failure, prior MI, prior percutaneous coronary intervention (PCI), prior coronary artery bypass grafting $(\mathrm{CABG})$, prior $\mathrm{AF} /$ flutter, implant type, pump time, cross-clamp time, STS score and LVEF. Greedy matching techniques without replacement and a caliper width equal to 0.2 of the standard deviation of the logit of the propensity score were applied to match male patients 1:1 to female patients. Standardized mean difference was used to evaluate the balance before and after matching. A standardized difference of 0.1 or less was deemed to be the ideal balance.

The paired t-test was used to compare the pre- and post-operative echocardiographic measurements and the absolute changes in LV size, LA size, and LA volume index between male and female patients. Cox proportional hazards regression models and the Fine \& Gray model [30] were implemented to determine the hazard ratios of sex difference on the primary and non-fatal secondary outcomes. McNemar test was performed to compare the postoperative complications by sex. The survival curve was plotted for all-cause mortality at longest follow-up using Kaplan-Meier methods. The interaction between sex and implant type for all-cause mortality at longest follow-up was tested by adding implant type and an interaction term of sex and implant type into the Cox model. Reverse Kaplan-Meier methods were used to estimate the median follow-up time. Statistical analyses were executed using the SAS 9.4. (SAS Institute, Cary NC). A p-value $<0.05$ was deemed of statistical significance. All statistical tests were two-sided.

\section{Results}

\section{Baseline Demographics}

The original database included 2,912 consecutive patients who had undergone MV surgery at the University of Alberta between January 1, 2004, and December 31, 2018 (Figure 1). 1,239 patients underwent an MVR and met the inclusion criteria. Baseline demographic data before and after propensity matching are summarized in Tables $1 \& 2$ respectively. Prior to propensity matching, males were found to be older than females, more likely to have renal disease, peripheral vascular disease, a prior MI, prior $\mathrm{CABG}$, an $\mathrm{LVEF}<30 \%$, and a higher STS risk score (Table 1). After propensity score matching, there were no significant differences in preoperative or intraoperative characteristics between females and males (Table 2).

\section{Surgical Detail}

Intraoperative differences before propensity matching included males having a longer time spent on cardiopulmonary bypass and a longer time with a cross-clamp placed (Table 1). These differences were not present after propensity matching. There were no significant differences in the types of valve replacements used with $79.8 \%$ of males and $77.2 \%$ of females receiving tissue valves (Table 1 ).

\section{Cardiac Remodelling}

Postoperative cardiac remodeling data are summarized in Tables 3 and 4 . Both groups showed a significant reduction in peak and mean MV gradients with males having an initial peak MV gradient of $18.5 \pm 10.2 \mathrm{mmHg}$ and a postoperative gradient of $15.1 \pm 6.4 \mathrm{mmHg}(\mathrm{p}=0.006)$. Females had an initial MV peak gradient of $20.7 \pm 9.4 \mathrm{mmHg}$ to $16 \pm 5.8 \mathrm{mmHg}$ postoperatively $(\mathrm{p}<0.001)$. Males began with a mean MV gradient from $8.6 \pm 6.8 \mathrm{mmHg}$ preoperatively to $5.7 \pm 2.9 \mathrm{mmHg}$ postoperatively $(\mathrm{p}=0.001)$, and females had an initial mean 
gradient of $9.2 \pm 5.1 \mathrm{mmHg}$ and a postoperative mean gradient of $5.7 \pm 2.8 \mathrm{mmHg}(\mathrm{p}<0.001)$. Both groups were found to have an increase in MVA after MVR with males having an initial MVA of $1.5 \pm 0.8 \mathrm{~cm}^{2}$ and a post replacement area of $1.8 \pm 0.9 \mathrm{~cm}^{2}(\mathrm{p}<0.001)$, and females with an initial MVA of $1.3 \pm 0.6 \mathrm{~cm}^{2}$ and a postoperative MVA of $1.8 \pm 0.9 \mathrm{~cm}^{2}(\mathrm{p}<0.001)$. Only males demonstrated significantly improved LV remodeling with an initial LVIDd of $54.7 \pm 8.9 \mathrm{~mm}$ and a postoperative LVIDd of $51.1 \pm 10.2 \mathrm{~mm}(\mathrm{p}<0.001)$. Initial LVIDd in females was $47.8 \pm 7.4 \mathrm{~mm}$, and postoperatively it was $47.2 \pm 7.7 \mathrm{~mm}(\mathrm{p}=0.251)$. Males also showed a significant reduction in LVEF with an initial LVEF of $51.9 \% \pm 10.6 \%$ and a postoperative LVEF of $49.2 \% \pm 11.9 \%$ ( $\mathrm{p}=0.003$ ). Females did not demonstrate the same reduction with an initial LVEF of $53.3 \% \pm 10.6 \%$ and a postoperative LVEF of $53.8 \% \pm 11.5 \%(\mathrm{p}=0.929)$. In contrast, it was only females that demonstrated significantly improved LA remodeling. Males had an initial LA volume index of $60 \pm 35.7 \mathrm{ml} / \mathrm{m}^{2}$ and a postoperative LA volume index of $60 \pm 28.8 \mathrm{ml} / \mathrm{m}^{2}(\mathrm{p}=0.077)$, while females began with a LA volume index from $57.9 \pm 24 \mathrm{ml} / \mathrm{m}^{2}$ preoperatively to $55.6 \pm 20.8 \mathrm{ml} / \mathrm{m}^{2}$ postoperatively $(\mathrm{p}=0.011)$. Females were also the only group to show a significant reduction in LA diameter. Males had an initial LA diameter of $5.4 \pm 1.2 \mathrm{~cm}$ and a postoperative diameter of $5.3 \pm 1.2 \mathrm{~cm}(\mathrm{p}=0.09)$. Females began with an LA diameter of $5.3 \pm 1.1 \mathrm{~cm}$ and post MV replacement had an LA diameter of $5 \pm 0.9 \mathrm{~cm}(\mathrm{p}=0.037)$. There were no significant differences in the absolute anatomical changes between males and females (Table 4$)$.

\section{Peri-operative Outcomes and Post-MVR Survival}

Secondary outcomes for these patients after propensity matching are summarized in tables 5 and 6 . There were no significant differences in mortality during the at 30 days, 1 year, or at longest follow-up. Although at all time points after 1 year, males had a numerically higher mortality rate than females (figure 2, table 5). At the median follow up time of 8.4 years, there were 107 deaths in the male group (57.5\%) and 99 deaths in the female group (50.9\%) [adjusted hazard ratio (HR): 1.19; 95\% confidence interval (CI): 0.9-1.56; $\mathrm{p}=0.22$. Males were more likely to experience an MI during the follow-up period with 20 MIs recorded (8.9\%) and 10 MIs recorded for the female group (5.5\%) [HR: 2.16; 95\% CI: 1.02-4.59; $\mathrm{p}=0.045]$. Males were also more likely to develop sepsis postoperatively. 28 males (8.9\%) and 11 females (3.5\%) developed sepsis postoperatively $(\mathrm{p}=0.005)$. There were no significant differences in the incidence of heart failure, readmission for stroke, reoperation of the MV, residual MR, new-onset $\mathrm{AF}$, pacemaker insertion rates, or acute kidney injury postoperatively.

\section{Conclusions}

In this study, we looked to identify the impact of sex on remodeling and outcomes post MVR. After propensity matching and isolating for surgical approach, our results demonstrate significant cardiac remodeling postoperatively for both sexes. Interestingly the cardiac remodeling was inconsistent between the two groups. Males showed significantly improved remodeling of the LV while females had significant remodeling of the LA. A study by Goldstein et al randomized 251 patients to either MV repair or replacement and followed the patients for 2 years. Both MV repair and replacement groups demonstrated significant remodeling of the LV end-systolic volume index within the first year postoperatively [22]. Ebd Alaziz \& Ibrahim similarly followed 20 males and 25 females who had undergone MVR for chronic MR. These patients demonstrated a reduction in LVEF from $61.09 \pm 7.6 \%$ to $59.67 \pm 6.56 \%$, in LA size from $5.55 \pm 0.88 \mathrm{~cm}$ to $4 \pm 0.54 \mathrm{~cm}$, and LV end-systolic diameter from $4.06 \pm 0.65 \mathrm{~cm}$ to $3.45 \pm 0.51 \mathrm{~cm}$ at 1-year postoperatively [23]. Even so, no studies have compared the differences in remodeling between sexes with a large number of propensity-matched patients and our results demonstrate a difference in the heart's ability to remodel after MVR. Males demonstrated a significant reduction in LV size and LVEF, while females did not. Females demonstrated a significant reduction in LA size and volume, while males did not. This distinction in cardiac remodeling demonstrates variability in cardiac adaptation after MV surgery. The significance of this difference has the potential to result in variable clinical outcomes for males and females, including long-term freedom from heart failure and mortality. Further study is necessary to fully elucidate this relationship.

In contrast to previous literature, our results demonstrated that after propensity matching and isolating for surgical approach females and males have similar rates of morbidity and mortality post MVR, and when there were significant differences between the sexes males had inferior outcomes. Vassileva et al performed a study 
including 47,602 patients undergoing isolated MV surgery and divided patients by sex and surgical approach [12]. This study found the MV repair rate for males was $44.0 \%$ and $31.9 \%$ for females, with remaining patients receiving MVR. Higher operative and in-hospital mortality rates were identified for females. Women demonstrated a higher mortality rate than men with $8.2 \%$ and $9.3 \%$ for males and females respectively. Even after adjustment for differences in baseline characteristics and admission status, the mortality rate for females undergoing MVR was still increased compared to their male counterparts [12]. Song et al demonstrated similar results with their study of 24,977 patients from The STS National Database who underwent isolated MV repair or replacement [18]. Females were shown to have significantly increased rates of morbidity and mortality with the largest difference being in the 40-59 year age group with approximately $2.5 \mathrm{x}$ the rates of mortality in females as compared to males, this survival disadvantage diminished with aging [18]. As there were multiple potential causes of these discrepancies in prior literature, we sought to identify whether sex alone impacts the outcomes post-MV surgery by matching patients based on comorbidities and isolating for surgical approach. We found no significant difference in mortality between males and females following MVR. Our results demonstrated that when males and females were matched for preoperative comorbidities and surgical approaches, males had inferior postoperative with an increased likelihood of developing sepsis and requiring rehospitalization due to MI postoperatively than females.

Our results have demonstrated significant variations in cardiac remodeling between males and females post MVR. Both males and females demonstrated significant changes in MVA as well as peak and mean gradients, although chamber remodeling was distinctly different between the sexes. Males demonstrated a significant reduction in LV diameter and LVEF while females did not show these changes. In contrast, females showed a significant reduction in LA diameter and volume while males did not. This difference in chamber remodeling demonstrates variability in the heart's ability to adapt after MV surgery. The significance of this difference is unknown but has the potential to result in different clinical outcomes between the sexes, including long-term freedom from symptoms of heart failure and rates of mortality. Further study of this discrepancy is necessary to fully elucidate this relationship.

\section{References}

1. Etzioni DA, Starnes VA. The epidemiology and economics of cardiothoracic surgery in the elderly. In: Katlic MR, editor. Cardiothoracic surgery in the elderly: evidence-based practice. New York: Springer 2011;5-24.

2. Benjamin EJ, Muntner P, Alonso, A, et al. Heart Disease and Stroke Statistics-2019 Update: A Report From the American Heart Association Circulation 2019;139:e56-e528.

3. Adamo M, Capodanno D, Cannata S, et al. Comparison of three contemporary surgical scores for predicting all-cause mortality of patients undergoing percutaneous mitral valve repair with the MitraClip system (from the multicenter GRASP-IT registry). American Journal of Cardiology 2015;115(1):107-112.

4. Kaartama T, Heikkinen L, Vento A. An evaluation of mitral valve procedures using the European system for cardiac operative risk evaluation. Scandinavian Journal of Surgery 2008;97(3):254-258.

5. Barili F, Pacini D, Grossi C, Di Bartolomeo R, Alamanni F, Parolari A. Reliability of new scores in predicting perioperative mortality after mitral valve surgery. Journal of Thoracic and Cardiovascular Surgery 2014;147(3):1008-1012.

6. Jamieson WR, Edwards FH, Schwartz M, Bero JW, Clark RE, Grover FL. Risk stratification for cardiac valve replacement. national cardiac surgery database. Database committee of the society of thoracic surgeons. The Annals of thoracic surgery 1999;67:943.

7. Wang TKM, Harmos S, Gamble GD, Ramanathan T, Ruygrok PN. Performance of contemporary surgical risk scores for mitral valve surgery. Journal of Cardiac Surgery 2017;32:172-6.

8. EUGenMed Cardiovascular Clinical Study Group, Regitz-Zagrosek V, Oertelt-Prigione S, et al. Gender in cardiovascular diseases: impact on clinical manifestations, management, and outcomes. European Heart Journal 2016;37(1):24-34. 
9. Saad M, Nairooz R, Pothineni NVK, et al. Long-Term Outcomes With Transcatheter Aortic Valve Replacement in Women Compared With Men: Evidence From a Meta-Analysis. Journal of the American College of Cardiology: Cardiovascular Interventions 2018;11(1):24-35.

10. Nishimura R, Otto C, Bonow R, et al. 2017 AHA/ACC focused update of the 2014 AHA/ACC guideline for the management of patients with valvular heart disease: A report of the american college of cardiology/american heart association task force on clinical practice guidelines. Circulation 2017;135:e1159-95.

11. Giustino G, Overbey J, Taylor D, et al. Sex-based differences in outcomes after mitral valve surgery for severe ischemic mitral regurgitation: From the cardiothoracic surgical trials network. Journal of the American College of Cardiology 2019;73:1951.

12. Vassileva CM, MD, McNeely C, BS, Mishkel G, MD, Boley T, MSN, Markwell S, MA, Hazelrigg S, MD. Gender differences in long-term survival of medicare beneficiaries undergoing mitral valve operations. Annals of Thoracic Surgery 2013;96:1367-73.

13. Vassileva CM, Stelle LM, Markwell S, Boley T, Hazelrigg S. Sex differences in procedure selection and outcomes of patients undergoing mitral valve surgery. The Heart Surgery Forum 2011;14(5):E276-E282.

14. Johnston A, Mesana TG, Lee DS, Eddeen AB, Sun LY. Sex Differences in Long-Term Survival After Major Cardiac Surgery: A Population-Based Cohort Study. Journal of the American Heart Association 2019;8(17):e013260.

15. Mokhles MM, Siregar S, Versteegh MI, et al. Male-female differences and survival in patients undergoing isolated mitral valve surgery: a nationwide cohort study in the Netherlands. European Journal of Cardiothoracic Surgery 2016;50(3):482-487.

16. Jung JC, Jang MJ, Hwang HY. Meta-Analysis Comparing Mitral Valve Repair Versus Replacement for Degenerative Mitral Regurgitation Across All Ages. American Journal of Cardiology 2019;123(3):446-453.

17. Yun-Dan D, Wen-Jing D, Xi-Jun X. Comparison of Outcomes following Mitral Valve Repair versus Replacement for Chronic Ischemic Mitral Regurgitation: A Meta-Analysis. The Journal of Thoracic and Cardiovascular Surgery 2017;65(6):432-441.

18. Song HK, Grab JD, O'Brien SM, Welke KF, Edwards F, Ungerleider RM. Gender differences in mortality after mitral valve operation: evidence for higher mortality in perimenopausal women. The Annuals of Thoracic Surgery 2008;85(6):2040-2045.

19. Gammie JS, Sheng S, Griffith BP, et al. Trends in mitral valve surgery in the united states: Results from the society of thoracic surgeons adult cardiac surgery database. The Annals of thoracic surgery 2009;87:1431.

20. Azevedo PS, Polegato BF, Minicucci MF, Paiva SAR, Zornoff LAM. Cardiac remodeling: Concepts, clinical impact, pathophysiological mechanisms and pharmacologic treatment. Arquivos brasileiros de cardiologia 2016;106:62-9.

21. Wu Q, Xiao Y, Yuan Y, et al. Mechanisms contributing to cardiac remodelling. Clinical science (1979) 2017;131:2319-45.

22. Goldstein D, Moskowitz AJ, Gelijns AC, et al. Two-Year Outcomes of Surgical Treatment of Severe Ischemic Mitral Regurgitation. N Engl J Med. 2016;374(4):344-353. doi:10.1056/NEJMoa1512913

23. Abd Elaziz ME, Ibrahim IM. Reversal of Left Ventricular Functions in Chronic Mitral Regurgitation after Mitral Valve Replacement. Heart Surg Forum. 2016;19(4):E160-E164.

24. Lazam S, Vanoverschelde JL, Tribouilloy C, Grigioni F, Suri RM, Avierinos JL, de Meester C, Barbieri A, Rusinaru D, Russo A, Pasquet A, Michelena H, Huebnner M, Maalouuff J, Clavel MA, Szymanski C, Enriquez-Sarano M. Twenty-Year Outcome after Mitral Repair Versus Replacement for Severe Degenerative Mitral Regurgitation. Analysis of a Large, Prospective, Multicenter International Registry. Circulation 2017;135(5):410-422. 
25. Mick SL, Keshavamurthy S, and Gillinov AM. Mitral valve repair versus replacement. Ann Cardiothorac Surg. 2015;4(3):230-237.

26. Hendrix RJ, Bello RA, Flahive JM, et al. Mitral Valve Repair Versus Replacement in Elderly With Degenerative Disease: Analysis of the STS Adult Cardiac Surgery Database. Ann Thorac Surg. 2019;107(3):747753. doi:10.1016/j.athoracsur.2018.09.018

27. Tran A, Ruel M, Chan V. Gender differences in outcomes following cardiac surgery: implications for managing patients with mitral valve disease. Current Opinion in Cardiology 2015;30(2):151-154.

28. Nagendran J, Nagendran J, Bozso SJ, et al. Coronary artery bypass surgery improves outcomes in patients with diabetes and Left Ventricular dysfunction. Journal of the American College of Cardiology 2018;71:819-27.

29. O'Brien SM, Feng L, He X, et al. The society of thoracic surgeons 2018 Adult cardiac surgery risk models: Part 2 - Statistical methods and Results. The Annals of Thoracic Surgery 18;105:1419-28.

30. Fine JP, Gray RJ. A proportional hazards model for the subdistribution of a competing risk. J Am Stat Assoc 1999;94:496-509.

31. Kislitsina ON, Zareba KM, Bonow RO, et al. Is mitral valve disease treated differently in men and women? European Journal of Preventive Cardiology 2019;26:1433-43.

32. McNeely C, Vassileva C. Mitral valve surgery in women: Another target for eradicating sex inequality. Circulation. Cardiovascular quality and outcomes 2016;9:S94-6.

Figure 1. Study Population Flowchart

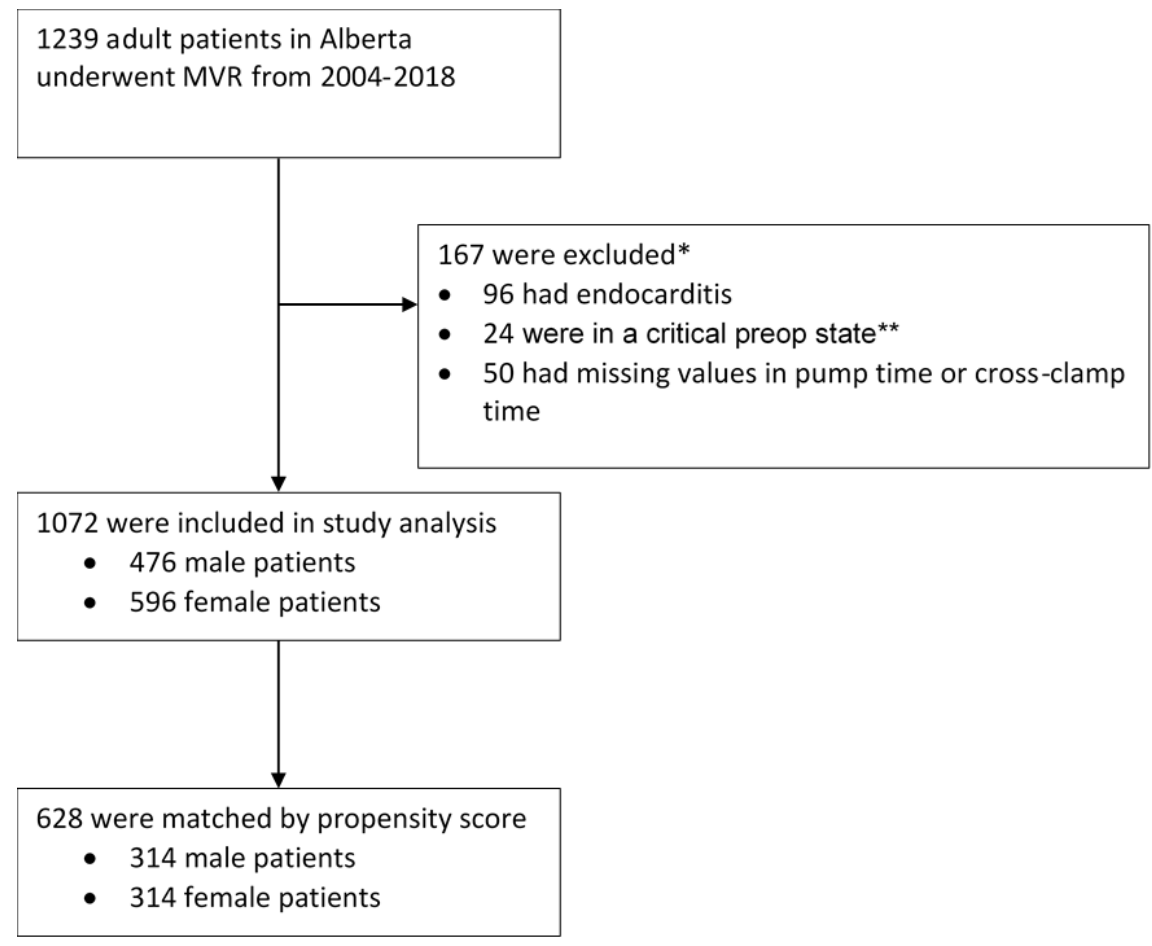

* Some overlap existed; therefore, the numbers breakdown did not add up to the total.

$* *$ Need for intra-aortic balloon pump, respiratory failure requiring vent, cardiogenic shock. 
Table 1. Baseline characteristics of patients that underwent MVR before matching $(\mathrm{N}=1072)$

\section{Hosted file}

image2.emf available at https://authorea.com/users/364532/articles/484917-impact-of-sex-oncardiac-remodelling-and-long-term-outcomes-following-mitral-valve-replacement

Values are No. (\%) or mean \pm SD

Table 2. Baseline characteristics of patients that underwent MVR after matching $(\mathrm{N}=628)$

\section{Hosted file}

image3.emf available at https://authorea.com/users/364532/articles/484917-impact-of-sex-oncardiac-remodelling-and-long-term-outcomes-following-mitral-valve-replacement

Table 3. Hemodynamic, Functional, and Anatomical Changes Before and After MVR(N=628)

\section{Hosted file}

image4.emf available at https://authorea.com/users/364532/articles/484917-impact-of-sex-oncardiac-remodelling-and-long-term-outcomes-following-mitral-valve-replacement

Table 4. Absolute anatomical changes before and after MVR(n=628).

\section{Hosted file}

image5.emf available at https://authorea.com/users/364532/articles/484917-impact-of-sex-oncardiac-remodelling-and-long-term-outcomes-following-mitral-valve-replacement

ECHO median follow-up time: 4 months.

Table 5. Secondary outcomes post MVR (Comparing male to female patients) (N=628).

\section{Hosted file}

image6.emf available at https://authorea.com/users/364532/articles/484917-impact-of-sex-oncardiac-remodelling-and-long-term-outcomes-following-mitral-valve-replacement

${ }^{*}$ The rates in brackets are estimates from Kaplan-Meier Curve.

Table 6. Post-operative complications post MVR ( $\mathrm{N}=628)$.

\section{Hosted file}

image7.emf available at https://authorea.com/users/364532/articles/484917-impact-of-sex-oncardiac-remodelling-and-long-term-outcomes-following-mitral-valve-replacement 


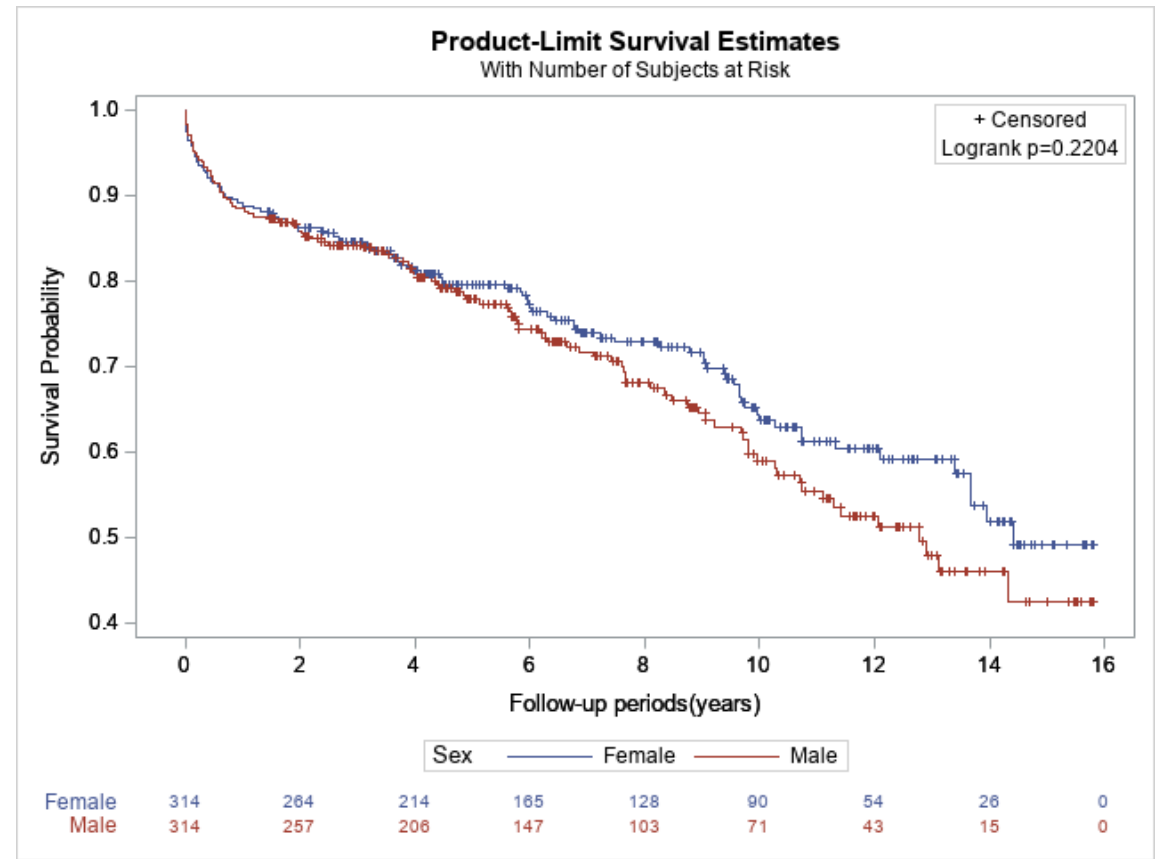

Figure 2. Kaplan Meier curve for all-cause mortality at longest available follow-up. 